\title{
Treatment of Café-au-lait Macules Using a 0-switched Laser Followed by Serial Fractional Thulium Laser Treatments
}

Nark-Kyoung Rho

Leaders Aesthetic Laser and Cosmetic Surgery Center, Seoul, Korea

Received May 22, 2017

Accepted May 25, 2017

\footnotetext{
Correspondence

Nark-Kyoung Rho

Leaders Aesthetic Laser and Cosmetic Surgery Center, THE CLASSIC500, 90 Neungdong-ro,

Gwangjin-gu, Seoul 05065, Korea

Tel.: +82-2-444-7585

Fax: +82-2-444-7535

E-mail: rhonarkahanmail.net

(C) Korean Society for Laser Medicine and Surgery

(c) This is an open access article distributed under the terms of the Creative Commons Attribution NonCommercial License (http://creativecommons.org/ licenses/by-nc/4.0) which permits unrestricted noncommercial use, distribution, and reproduction in any medium, provided the original work is properly cited.
}

Café-au-lait macules are a benign cutaneous pigmentary disorder with variable responses to laser treatment. We treated a 21 -year-old female with recurred café-au-lait macules on the thigh using a combination of frequency-doubled Q-switched neodymium-doped yttrium aluminum garnet laser followed by 10 sessions of fractional laser, via a novel 1,927 $\mathrm{nm}$ fractional thulium laser. At the 11-month follow-up after the last treatment, no foci of re-pigmentation were found. We suggest that fractional thulium laser resurfacing, combined with conventional Q-switched laser treatment, can be used as a safe and effective adjuvant therapy in patients with treatment-resistant café-au-lait macules.

\section{Key words}

Café-au-lait macule; Fractional laser; Q-switched laser; Thulium laser 


\section{INTRODUCTION}

The removal of café-au-lait macules (CALMs) are related with a significant challenge due to variable response to therapeutic options, high rate of recurrence and increased risk of adverse effects. We report the use of a novel fractional thulium laser system for the treatment of CALMs which recurred after a conventional $Q$-switched laser treatment.

\section{CASE REPORT}

A 21-year-old healthy Korean female presented to our clinic for removal of a pigmented lesion on the anterior thigh, which had been present since childhood. According to the patient, the lesion had been previously treated by "a pigment laser" but relapsed after several months. Physical examination revealed variably pigmented brown patches with irregular shapes and borders on the anterior side of left thigh (Fig. 1A). Under the clinical diagnosis of recurred CALMs, she received treatment using a frequency-doubled Q-switched neodymium-doped yttrium aluminum garnet (Nd:YAG) laser (Con-Bio RevLite SI; Cynosure, Inc., Westford, MA, USA) with the following parameter: 532 $\mathrm{nm}, 2 \mathrm{~mm}$ spot, $3.0 \mathrm{~J} / \mathrm{cm}^{2}, 1$ pass with minimal overlap). She demonstrated near complete clinical improvement at the follow-up 4 months after the Q-switched laser treatment (Fig. 1B). Eleven months after the Q-switched laser treatment, the patient visited our clinic with the recurrence of pigments. Diffuse, irregular pigmented patches were noted on the previous laser treatment site (Fig. 1C). The patient's pigmentary patches were treated using a novel 1,927-nm fractional thulium-doped fiber laser (LASEMDTM; Lutronic Corp., Goyang, Korea). Each pulse of this laser system generates 40 fractionated zones of thermal injury over an area of about $10 \mathrm{~mm} \times 4$ $\mathrm{mm}$. After fractional irradiation with treatment Level 3 (5 W, $2.4 \mathrm{~mJ}$ ) and 5-6 laser passes, manufacturer-supplied vitamin $\mathrm{C}$-containing hydrogel was applied to the treatment area. Ten treatment sessions were performed with an interval of 2-3 weeks. Treatment was well-tolerated with the use of topical anesthetic cream application before laser irradiation. Temporary erythema was noted for 2 days and microcrusts existed for one week after laser treatment. Besides, no significant adverse events were noted throughout the treatment period. The patient was followed up at 6 months after the laser fractional thulium laser treatment and there were no signs of recurrence of CALMs (Fig. 1D).

\section{DISCUSSION}

The most frequently used lasers for the treatment of CALMs are the $\mathrm{Q}$-switched lasers, including ruby, ${ }^{1}$ $\mathrm{Nd}: Y A G,{ }^{2}$ and alexandrite. ${ }^{3}$ Other approaches using erbium:YAG laser ${ }^{4}$ or low-fluence Q-switched laser ${ }^{5}$ have been reported for the treatment of CALM. However, the results are inconsistent and the therapeutic outcome is not easy to predict in general. ${ }^{2}$ Recently, the use of fractional laser systems, either ablative ${ }^{6}$ or non-ablative, ${ }^{7}$ have been reported in the literature. In the present case, previous relapse of CALMs may have partially resulted from the age of the patient and anatomic site of treatment, as the lower extremity is known to yield the poor response to laser treatment. To the best of our knowledge, this is the first report using fractional thulium laser resurfacing
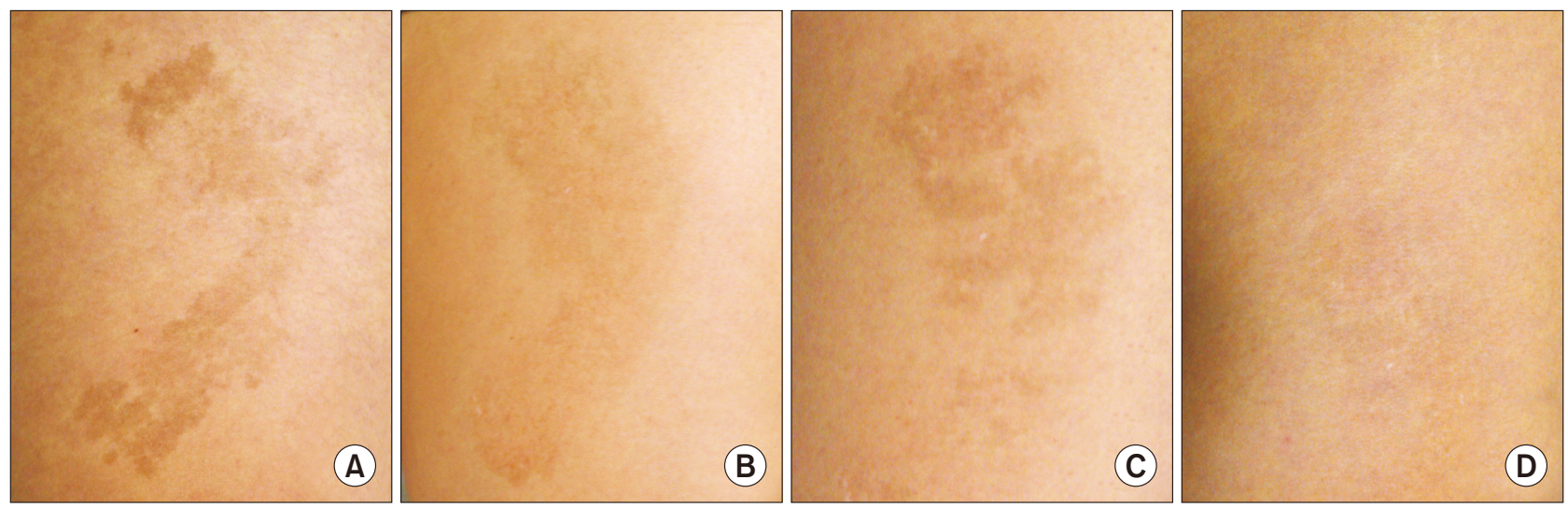

Fig. 1. Serial photograph of a 21-year-old female with recurred café-au-lait macules on anterior thigh (A), demonstrating near-complete resolution (B, 4 months) and re-pigmentation (C, 11 months) after the Q-switched laser treatment. Six months after 10 sessions of fractional thulium laser resurfacing, the lesion was clear with no visible signs of recurrence (D). 
for the treatment of therapy-resistant CALMs. The novel wavelength of 1,927 $\mathrm{nm}$ delivered by the thulium laser has been studied for the treatment of photoaging and melasma. ${ }^{8}$ This wavelength has a higher absorption coefficient for water than the conventional fractional $1550 \mathrm{~nm}$ lasers, conferring a greater ability to target epidermal structures with precision with minimal damage to the dermis. The other characteristic of this wavelength is its reported maximum depth of penetration of $200 \mu \mathrm{m}$. This superficial treatment depth, along with its high water affinity, makes thulium be very suitable for epidermal pigmentary indications. Because the pathology in café-au-lait macules is located entirely within the basal layer of the epidermis, some authors ${ }^{4}$ suggest that atraumatic removal of the epidermis with ablative lasers might be useful. The use of fractional thulium laser is advantageous over conventional ablative laser resurfacing treatments which have drawbacks including pain and downtime. However, due to its fractional treatment pattern, this approach should be performed repeatedly. Considering most recurrences of CALMs occur within 1 year of treatment and our follow up showing no repigmentation has only been for 6 months, it remains unknown whether treatment permanently resolved the patient's conditions. However, we suggest that repetitive fractional thulium laser irradiation is effective, safe, and well-tolerated treatment modality with no postinflammatory hyperpigmentation and minimal downtime. Further long-term, controlled studies are recommended to validate the efficacy of this novel fractional laser system.

\section{REFERENCES}

1. Shimbashi T, Kamide R, Hashimoto T. Long-term follow-up in treatment of solar lentigo and café-au-lait macules with Q-switched ruby laser. Aesthetic Plast Surg 1997;21:445-8.

2. Levy JL, Mordon S, Pizzi-Anselme M. Treatment of individual café au lait macules with the Q-switched Nd:YAG: a clinicopathologic correlation. J Cutan Laser Ther 1999;1:21723.

3. Wang Y, Qian H, Lu Z. Treatment of café au lait macules in Chinese patients with a Q-switched 755-nm alexandrite laser. J Dermatolog Treat 2012;23:431-6.

4. Alora MB, Arndt KA. Treatment of a café-au-lait macule with the erbium:YAG laser. J Am Acad Dermatol 2001;45:566-8.

5. Kim HR, Ha JM, Park MS, Lee Y, Seo YJ, Kim CD, et al. A lowfluence 1064-nm Q-switched neodymium-doped yttrium aluminium garnet laser for the treatment of café-au-lait macules. J Am Acad Dermatol 2015;73:477-83.

6. Chung BY, Han SS, Moon HR, Lee MW, Chang SE. Treatment with the pinhole technique using erbium-doped yttrium aluminium garnet laser for a café au lait macule and carbon dioxide laser for facial telangiectasia. Ann Dermatol 2014;26: 657-9.

7. Balaraman B, Ravanfar-Jordan P, Friedman PM. Novel use of non-ablative fractional photothermolysis for café-au-lait macules in darker skin types. Lasers Surg Med 2017;49:84-7.

8. Lee HM, Haw S, Kim JK, Chang SE, Lee MW. Split-face study using a 1,927-nm thulium fiber fractional laser to treat photoaging and melasma in Asian skin. Dermatol Surg 2013;39:879-88. 